\title{
Lentviral-mediated RNAi to inhibit target gene expression of the porcine integrin $\alpha v$ subunit, the FMDV receptor, and against FMDV infection in PK-15 cells
}

\author{
Jihuai Luo, Junzheng Du, Shandian Gao, Guofeng Zhang, Jingjing Sun, Guozheng Cong, Junjun Shao, Tong Lin \\ and Huiyun Chang*
}

\begin{abstract}
Background: shRNA targeting the integrin $\alpha$ v subunit, which is the foot-and-mouth disease virus (FMDV) receptor, plays a key role in virus attachment to susceptible cells. We constructed a RNAi lentiviral vector, iav pLenti6/BLOCK -iTTM, which expressed siRNA targeting the FMDV receptor, the porcine integrin $\alpha$ v subunit, on PK-15 cells. We also produced a lentiviral stock, established an iav-PK-15 cell line, evaluated the gene silencing efficiency of mRNA using real-time qRT$P C R$, integrand $\alpha v$ expression by indirect immunofluorescence assay (IIF) and cell enzyme linked immunosorbent assays (cell ELISA), and investigated the in vivo inhibitory effect of shRNA on FMDV replication in PK-15 cells.
\end{abstract}

Results: Our results indicated successful establishment of the i $\alpha \vee$ U6 RNAi entry vector and the i $\alpha \vee$ pLenti6/BLOCK -iT expression vector. The functional titer of obtained virus was $1.0 \times 10^{6} \mathrm{TU} / \mathrm{mL}$. To compare with the control and mock group, the iav-PK-15 group av mRNA expression rate in group was reduced by $89.5 \%$, whilst IIF and cell ELISA clearly indicated suppression in the experimental group. Thus, iav-PK-15 cells could reduce virus growth by more than three-fold and there was a > 99\% reduction in virus titer when cells were challenged with $10^{2} \mathrm{TCID}_{50}$ of FMDV.

Conclusions: lav-PK-15 cells were demonstrated as a cell model for anti-FMDV potency testing, and this study suggests that shRNA could be a viable therapeutic approach for controlling the severity of FMD infection and spread.

\section{Background}

Foot-and-mouth disease virus (FMDV) is a picornavirus, which causes a highly contagious disease in clovenhoofed animals. Foot-and-mouth disease (FMD) is a threat to the worldwide livestock industry, because FMD can have a devastating effect on a country's economy. FMDV shows a high genetic and antigenic variability, which is reflected in the seven serotypes and numerous variants described to date [1]. The FMDV genome is composed of a positive-sense single-stranded RNA molecule of about 8500 nucleotides, which contains a unique open reading frame. There are seven distinguishable serological types, i.e., O, A, C, Asia1, SAT1, SAT2, and SAT3, and more than 65 subtypes.

\footnotetext{
* Correspondence: changhuiyun@126.com

State Key Laboratory of Veterinary Etiological Biology, National Foot and Mouth Disease Reference Laboratory, Lanzhou Veterinary Research Institute, Chinese Academy of Agricultural Sciences, Lanzhou 730046, China
}

Recently, RNAi has shown promise as a therapeutic in many infections, including, viral diseases of animals and humans. RNAi is a process of sequence specific, posttranscriptional gene silencing (PTGS) in animals and plants, which is conducted using 21 to 33 nucleotides (nt) of small interfering RNA (siRNA), which are homologous in sequence to the silenced gene [2]. In eukaryotic organisms, dsRNA is produced in vivo or introduced by pathogens and processed into 21-23 nucleotide doublestranded short interfering RNA duplexes (siRNA) using an enzyme called Dicer, which is a member of the RNase III family of double-stranded RNA-specific endonucleases $[3,4]$. Each siRNA ia then incorporated into an RNA-induced silencing complex (RISC), which is an enzyme complex used to target cellular transcripts complementary to the siRNA for specific cleavage and degradation $[5,6]$. In addition to dsRNA, other endogenous RNA molecules have been shown to be capable of 
triggering gene silencing, including, short temporal RNA (stRNA) and microRNA (miRNA) [7].

RNAi has been widely studied as an antiviral technology for combating FMDV [8,9]. FMDV is antigenically variable, undergoing rapid mutation, which allows it to easily escape the host immune system via the high variability of its surface antigens. Existing vaccines and antiviral drugs have limited effectiveness, so the development of new strategies is essential. High genetic variability is a major issue that must be addressed in order to establish RNAi as a viable approach against FMDV [10]. Current measures for the control of FMDV replication include, RNAi plasmid 1D or 3D [11], VP1 [9], IRES [12], all of which are focused on several regions of the FMDV genome [13]. RNAi directed at specific gene sequences of certain FMDV strains might be a risky strategy, especially in the event of an emergent FMD outbreak in the absence of information concerning the serotype or genotype of the isolated pathogen, although early protection is needed. The integrin $\alpha \mathrm{v}$ subunit is the FMDV receptor for each serotype or genotype and the integrin $\alpha \mathrm{v}$ subunit of the FMDV receptor plays a key role in the attachment of the virus to susceptible cells. Studies have shown a clear correlation between sensitivity of FMDV and expression of integrins $\alpha v \beta 1, \alpha v \beta 3, \alpha v \beta 6$, and $\alpha v \beta 8$. Most integrin subunits have independent functions and are essential for normal development [14,15]. $\alpha 1$-null mice are viable, fertile and apparently normal, whereas $\alpha 7$ null mice develop muscular dystrophy, and $\alpha 9$-null mice die within 10 day of birth. Mutations in many of the ligands for $\alpha v$ integrins are also viable. Thus, although many of these ligands and $\alpha \mathrm{v}$ integrins are widely expressed during development, they do not appear to be essential $[16,17]$. In this study, we used "knockdown" rather than "knockout" target gene expression of the porcine integrin $\alpha \mathrm{v}$ subunit, the FMDV receptor, to establish a cell line that could inhibit FMDV replication. Therefore, our work on RNAi with FMDV is a step forward on all previous work, particularly with respect to the cell model system.

In this study, we constructed an RNAi lentiviral vector that inhibited target gene expression of the porcine integrin $\alpha \mathrm{v}$ subunit and the FMDV receptor in PK-15, and FMDV replication in i $\alpha \mathrm{v}-\mathrm{PK}-15$. The vector was capable of inhibiting viral replication in cultured porcine PK-15 cells.

\section{Methods \\ Cells and viruses}

293T cells and PK-15 cells were cultured in Dulbecco's modified Eagle's medium (DMEM, GIBCO ${ }^{\mathrm{TM}}$, Invitrogen Corporation, Grand Island, NY, USA) supplemented with $10 \%$ heat-inactivated fetal bovine serum (FBS) $(\mathrm{pH}$ 7.4). Cultures were incubated at $37^{\circ} \mathrm{C}$ with $5 \% \mathrm{CO}_{2}$.
FMDV O/CHA/99 [GenBank accession number AF506822] was used for viral challenge.

\section{Construction of Lentivirus vector and establishment of the i $\alpha v-P K-15$ cell line}

Double-stranded oligo [18] (ds oligo)

5'-CACCGGACGGAACAAAGACTGTTGACGAAT CAACAGTCTTTGTTCCGTCC

CCTGCCTTGTTTCTGACAACTGCTTAGTTGTCAGAAACAAGGCAGGAAAA-3'

was chemically designed and synthesized by the Invitrogen Corporation, and the ds oligo was cloned into the linearized $\mathrm{pENTR}^{\mathrm{TM}} / \mathrm{U} 6$ vector. The $\mathrm{pENTR}{ }^{\mathrm{TM}} / \mathrm{U} 6$ entry vector provides a rapid and efficient way to clone oligo duplexes encoding a desired shRNA target sequence into a vector containing an RNA Pol III-dependent expression cassette for use in RNAi analysis. We generate an i $\alpha \mathrm{v}-$ pLenti6/BLOCK-iTTM expression clone construct using an LR recombination reaction between the i $\alpha \mathrm{v}-\mathrm{pENTR}^{\mathrm{TM}} / \mathrm{U} 6$ entry clone and the pLenti6/BLOCK-iT'TM-DEST vector (Figure 1). We used the expression clone construct and the LR clonase enzyme mix to produce a lentiviral construct. We co-transfected the 293FT producer cell line with three optimized packaging plasmids (pLP1, pLP2,

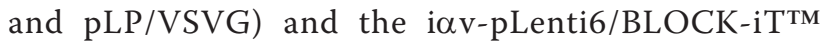
expression clone construct, which resulted in production of lentiviral stocks with a suitable titer. Stably transduced PK-15 cells were selected using blasticidin, with the minimum concentration of blasticidin required to kill untransduced PK-15 cells. The following procedure was used to determine the titer of the lentiviral stock using the PK-15 cells. To set up the stable cell line selection, we first needed to produce a lentiviral stock ( $\mathrm{i} \alpha \mathrm{v}-\mathrm{PK}-15$ containing the packaged i $\alpha v$ pLenti6/BLOCK-iTTM-DEST expression construct and a control containing the packaged pLenti6/ BLOCK-iTTM-DEST expression construct) by co-transfecting the optimized ViraPower ${ }^{\mathrm{TM}}$ packaging mix or pLenti6/ BLOCK-iT ${ }^{\mathrm{TM}}$-DEST expression construct into the 293FT producer cell line. PK-15 cell cultures were incubated at $37^{\circ} \mathrm{C}$ with $5 \% \mathrm{CO}_{2}$. The day before transduction, cells were trypsinized, diluted with DMEM fresh medium, and seeded into 6-well culture plates. On the day of transduction (day one), when PK- 15 cells were about $80 \%$ confluent, we thawed the lentiviral stock and diluted the appropriate amount of virus $(\mathrm{MOI}=5)$ into fresh DMEM complete medium. The culture medium was removed from the cells. The medium containing virus was gently mixed by pipetting and added to the cells. Polybrene was added to a final concentration of $6 \mu \mathrm{g} / \mathrm{mL}$. Cells were incubated for approximately six hours prior to changing the medium. The following day (day two), the medium containing virus was removed and replaced with fresh, complete culture medium. The following day (day three), 
5- CACCGGACGGAACAAAGACTGTTGACGAATCAACAGTCTTTGTTCCGTCC

CCTGCCTTGTTTCTGACAACTGCTTAGTTGTCAGAAACAAGGCAGGAAAA-3

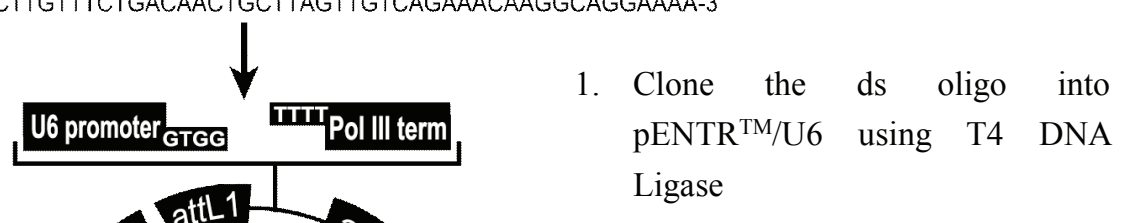

2. Transform E. coli and analyze colonies for the desired entry clone.

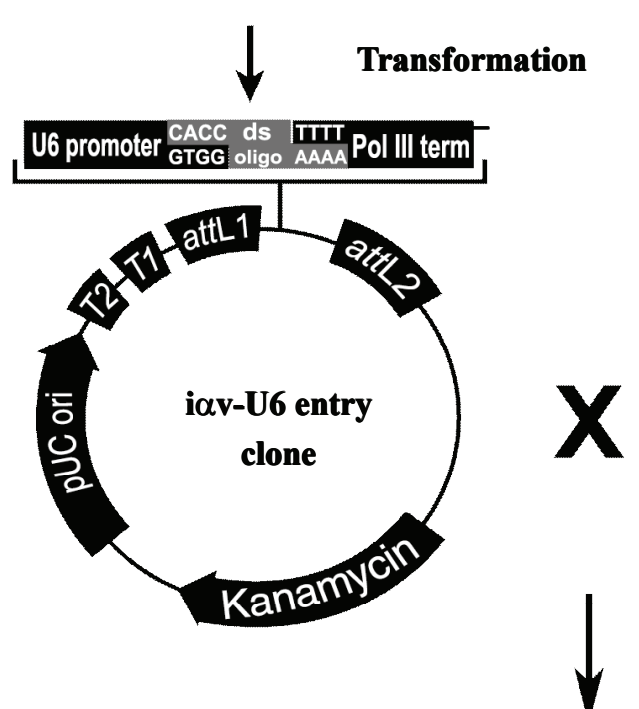
clone.

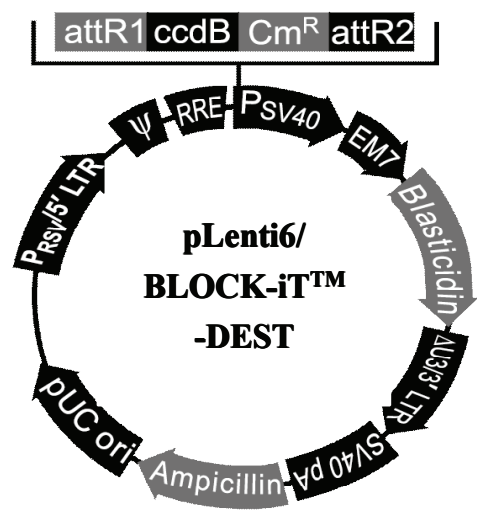

3. Perform and LR recombination reaction between the $\mathrm{pENTR}^{\mathrm{TM}}$ /U6 entry construct and pLenti6 /BLOCK-iT ${ }^{\mathrm{TM}}$-DEST generate the iav-pLenti6/BLOCK-iT Expression Construct.

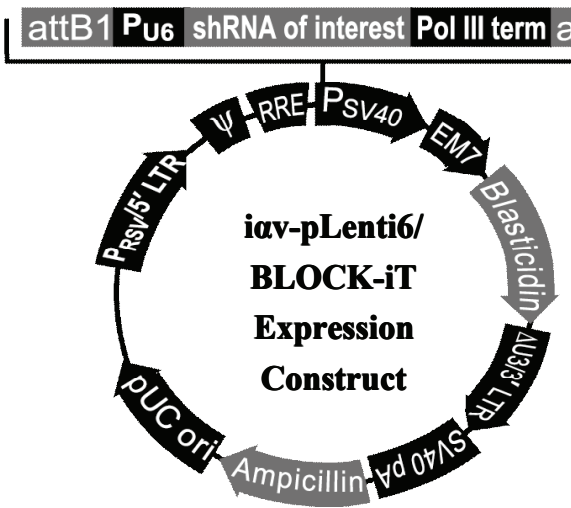

Figure 1 Construction of i $\alpha$ v-pLenti6/BLOCK-iT Expression Construct.

the medium was again removed and replaced with fresh, complete medium containing the appropriate amount of 2 $\mu \mathrm{g} / \mathrm{mL}$ blasticidin to select for stably transduced cells. Selection took up to two weeks. At some point there was a massive cell death and most of the cells were washed from the bottom of the dish, leaving colonies of stable cells behind. Blasticidin-resistant colonies were picked using a Gilson pipette with a sterile tip, by lowering the tip to the surface of the colony of interest then scraping and sucking gently until they were pulled into the tip. Colonies were 
transferred to a well in a 24-well plate and the process was repeated with other colonies. When wells were confluent, they were split into one well of a 6 -well plate (passage number 20) with a lower amount of blasticidin for maintenance $(1 \mu \mathrm{g} / \mathrm{mL})$. Cells were then assayed for knockdown of the target gene in one of the clones.

\section{Assay for knockdown of the i $\alpha \mathrm{v}-\mathrm{PK}-15$ cells RNA isolation and real-time qRT-PCR [19]}

Targeted gene expression was detected by establishing i $\alpha \mathrm{v}$-PK-15 cell lines at passage number 20 , which were cultured in DMEM supplemented with $10 \%$ heat inactivated FBS, and total RNA was extracted from i $\alpha v-P K-15$ cell cultures using an RNeasy Mini Kit (Invitrogen), before real-time qRT-PCR analysis using the SYBP RTPCR Kit (TaKaRa, Japan). Specific amplification was confirmed by melting curve analysis of the real-time qRT-PCR products, performed according to the manufacturer's protocol. Fluorescence was measured after each cycle and displayed graphically with a Stratagene Real time PCR Mx3000p (USA). Beta actin was used as a positive control and the $\alpha \mathrm{v}$ cDNA was amplified from cDNA preparations of i $\alpha \mathrm{v}-\mathrm{PK}-15$ cells by PCR using the primers shown in Table 1. The PCR procedures followed the manufacturer's instruction, with the following thermal profile: $10 \mathrm{~s}$ at $95^{\circ} \mathrm{C}$ for predenaturation; 40 cycles of $5 \mathrm{~s}$ at $95^{\circ} \mathrm{C}$; and $20 \mathrm{~s}$ at different annealing temperatures, depending on the integrins that were amplified. The specific amplification was confirmed by melting curve analysis of the real-time qRT-PCR products according to the manufacturer's protocol, with the following thermal profile: 1 cycle of $95^{\circ} \mathrm{C}$ for $60 \mathrm{~s} ; 55^{\circ} \mathrm{C}$ for $30 \mathrm{~s}$; and $95^{\circ} \mathrm{C}$ for $30 \mathrm{~s}$. PCR products were run on $3 \%$ agarose gel containing ethidium bromide and DNA bands were visualized using a UV transilluminator. IIF

Indirect immunofluorescence (IIF) assay was carried out to detect expression of the targeted $\alpha \mathrm{v}$ protein on the cell surface of an established i $\alpha \mathrm{v}-\mathrm{PK}-15$ cell line at passage number 20, which was cultured in DMEM supplemented with $10 \%$ heat inactivated FBS. Cells were grown on acid-washed cover slips to ensure adherence of cells. The culture media was carefully aspirated from the culture dish using a pipette, because vacuum aspiration

Table 1 Primer sequences used in this study

\begin{tabular}{llll}
\hline Primer sequence & Subunit & Size & Purpose \\
\hline AF 5'-GATTGTTGTTACTGGCTGTTTTGG-3' & $\alpha v$ & $94 \mathrm{bp}$ & $\begin{array}{l}\text { Real-time } \\
\text { PCR }\end{array}$ \\
\hline AR 5'-TGTTCCCTTTCCTGTTCTTCTTG-3' & & & \\
\hline ACTF 5'-GGACTTCGAGCAGGAGATGG-3' & B-actin & $138 \mathrm{bp}$ & $\begin{array}{l}\text { Real-time } \\
\text { PCR }\end{array}$ \\
\hline
\end{tabular}

ACTR 5'-AGGAAGGAGGGCTGGAAGAG-3' might also suck up cells from the cover slip surface. Cells were fixed in $4 \%$ paraformaldehyde (PFA) for $20 \mathrm{~min}$ at room temperature and were permeabilized for $5 \mathrm{~min}$ with $0.1 \%$ triton X-100 in $1 \times$ PBS at room temperature. Cells were then incubated in freshly prepared $50 \mathrm{mM}$ $\mathrm{NH}_{4} \mathrm{Cl}$ for 15 min to quench free aldehyde groups from PFA, before fixation after washing three times with $1 \times$ PBS. Cultures were blocked with $1 \%$ BSA in $1 \times$ PBS for 30 minutes at room temperature, before incubation with primary antibody diluted in blocking buffer (1\% BSA in PBS) for $1 \mathrm{~h}$ at room temperature. After primary incubation, cover slips were returned to the dishes and washed in PBS five to six times, for $5 \mathrm{~min}$ each wash. Secondary incubation was for $1 \mathrm{~h}$ at room temperature. The diluted antibody was centrifuged before use to pellet aggregates and covered in foil to protect from light. A control was included, which was comprised of the secondary antibody alone. The cover slips were returned to the dish and washed five times, for 5 min each wash using PBS, ensuring that samples were protected from the light. Cover slips were mounted by dipping the cover slips in distilled water to remove salt from PBS, before blotting the edge using filter paper to dry off excess water, before placing the cover slip cell-side down on a drop of mounting medium on a glass slide. The mounting medium used was DAPI (USA). The cover slip was stabilized on the slide using nail lacquer and left to dry overnight before viewing.

\section{Indirect cell ELISA for adherent Cells}

We added $100 \mu \mathrm{L}$ of the cell suspension $\left(1.0 \times 10^{5}\right.$ cells in each well of a 96-well culture plate) and incubated in 5\% $\mathrm{CO}_{2}$ at $37^{\circ} \mathrm{C}$, for at least $24 \mathrm{~h}$ allowing cells to attach. We removed the residual culture medium by inversion and by gently tapping the inverted plate on a paper towel. We dispensed the primary antibody $(50 \mu \mathrm{L})$, which was diluted in ice-cold ELISA buffer at the optimal concentration, whilst holding the pipette tip against the walls of the wells, then incubated for $1 \mathrm{~h}$ at $4^{\circ} \mathrm{C}$. The unbound primary antibody was removed by vacuum aspiration, whilst taking care to avoid drying. The plate was washed by gently adding $200 \mu \mathrm{L}$ of ice-cold washing buffer, whilst holding the pipette tip against the wall of the well to prevent detachment and loss of cells. The washing step was repeated four more times. After the last washing, the washing buffer was removed by inversion, whilst gently tapping the inverted plate on a paper towel. We then added the HRP-conjugated to the specific antibody for the primary antibody $(50 \mu \mathrm{L})$ diluted in ice-cold ELISA buffer at the optimal concentration before incubating the plate for $1 \mathrm{~h}$ at $4^{\circ} \mathrm{C}$. After five washings with PBS containing 1\% BSA, $100 \mu \mathrm{L}$ of tetramethylbenzidine (TMB) substrate was added to each well and incubation was continued for an additional $10 \mathrm{~min}$ at room temperature. We stopped the enzyme reaction by adding $50 \mu \mathrm{L}$ of $1.25 \mathrm{M} \mathrm{H}_{2} \mathrm{SO}_{4}$, and the optical 
density (OD) was measured with an ELISA reader (iEMSReader; Labsystems, Helsinki, Finland) at $A_{450 \mathrm{~nm}}$.

Viral challenge assay for the iov-PK-15 cells

Virus infectivity was determined by serial dilution of cell samples grown in 96-well plates. The virus titer was calculated as the $50 \%$ tissue culture infective dose $\left(\mathrm{TCID}_{50}\right)$ using the Reed-Muench formula [20]. A viral suspension titrated at $10^{4.41} \mathrm{TCID}_{50}$ was used in the experiment. Monolayers (about 50\% confluent) were grown in 96-well plates to assess the capacity of viruses to grow in PK-15 cells expressing siRNAs. Cells in one well of the 96-well plates were infected with $100 \mathrm{TCID}_{50}$ of virus per $0.1 \mathrm{ml}$. After $1 \mathrm{~h}$ of adsorption, the inoculum was removed and cells were washed twice with DMEM. The infection then proceeded in DMEM supplemented with no-fetal bovine serum and the virus titers were determined after two days. Statistical analysis was performed with the Microsoft Excel program.

\section{Results}

Target gene expression of the porcine integrin $\alpha \mathrm{v}$ subunit (the FMDV receptor) constructed with an RNAi lentivirus vector and established as an i $\alpha \mathrm{v}-\mathrm{PK}-15$ cell line We constructed the i $\alpha \mathrm{v}-$ pENTR ${ }^{\mathrm{TM}} / \mathrm{U} 6$ entry clone and i $\alpha v-p L e n t i 6 / B L O C K-i T^{\mathrm{TM}}$ expression clone by producing a lentiviral stock (containing the packaged i $\alpha \mathrm{v}$ pLenti6/BLOCK-iT ${ }^{\mathrm{TM}}$-DEST expression construct, or the packaged pLenti6/BLOCK-iT ${ }^{\mathrm{TM}}$-DEST expression construct) and co-transfecting the optimized ViraPower ${ }^{\mathrm{TM}}$ Packaging Mix $(3.0 \mu \mathrm{g} / \mu \mathrm{l})$ and i $\alpha \mathrm{v}$-pLenti6/ BLOCK-iT ${ }^{\mathrm{TM}}$-DEST expression construct or pLenti6/ BLOCK-iT $\mathrm{T}^{\mathrm{TM}}-\mathrm{DEST}$ expression construct $(3 \mu \mathrm{g})$ into the 293FT producer cell line, then harvested the viral supernatant, and determined the lentiviral stock titer with blasticidin. We picked blasticidin-resistant clones and cultured then in DMEM supplemented with $10 \%$ heatinactivated FBS at passage number 20. The sequencing results demonstrated successful cloning of the i $\alpha \mathrm{v}$ pENTR $^{\mathrm{TM}} / \mathrm{U} 6$ entry clone and the i $\alpha \mathrm{v}$-pLenti6/BLOCK $-\mathrm{i} \mathrm{T}^{\mathrm{TM}}$ expression clone construct. The functional titer of the virus obtained was $1.0 \times 10^{6} \mathrm{TU} / \mathrm{mL}$. We established stabled iav-PK-15 cells line at passage number 20 , which inhibited target gene expression in the porcine integrin $\alpha \mathrm{v}$ subunit, the FMDV receptor.

Real-time qRT- PCR assay of inhibition of RNA replication To demonstrate the level of inhibition, i $\alpha \mathrm{v}-\mathrm{PK}-15$ cells were collected at passage number 20 and real-time qRTPCR analysis was performed (Figure 2). The level of the $\alpha v$ gene was determined by real-time qRT-PCR and significantly decreased by about $89.5 \%$ in i $\alpha \mathrm{v}-\mathrm{PK}-15$ cells. Out results indicate that i $\alpha \mathrm{v}-\mathrm{PK}-15$ cells can effectively and specifically target gene expression of the porcine integrin $\alpha v$ subunit, the FMDV receptor.

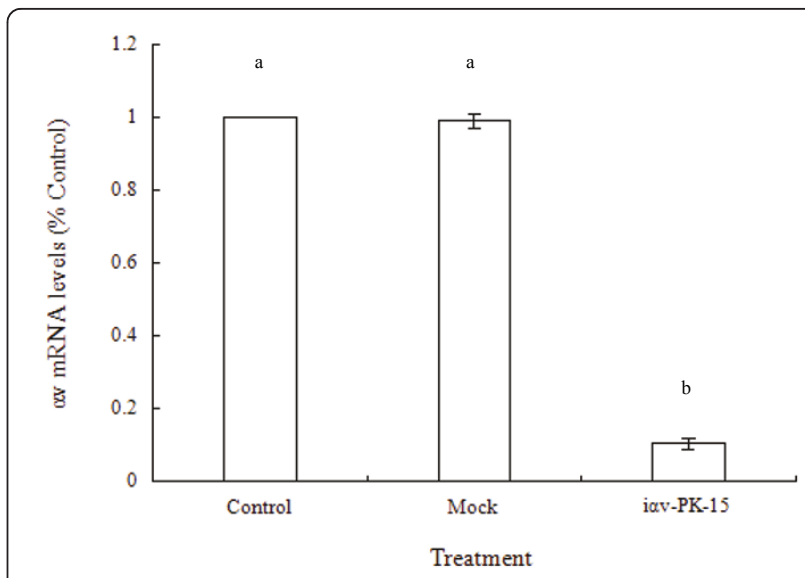

Figure 2 Downregulation of $\alpha v$ mRNA of i $\alpha v-P K-15$ detected with real-time qRT-PCR. Relative mRNA levels of $\alpha v$ gene are presented as the ratio of the $\alpha \vee$ mRNA quantity and the $1 \mathrm{ng} \beta$ actin (ng/1 ng $\beta$-actin) to facilitate comparison with the levels of inhibition of the $\alpha v$ gene. Data are shown as the average of three repeated experiments. Error bars show standard deviations. There was a highly significant difference between the control and mock, and i $\alpha$ v-PK-15 $(P<0.01)$

Level of inhibition of integrin $\alpha v$ subunit expression by IIF and indirect cell ELISA for adherent cells

Indirect immunofluorescence was used to visualize the cells under a fluorescence microscope. Control and mock exhibited a lot of green fluorescence, whereas significant reduction was observed in the green fluorescent signal of i $\alpha \mathrm{v}-\mathrm{PK}-15$, relative to the control and mock, indicating that $\mathrm{i} \alpha \mathrm{v}-\mathrm{PK}-15$ cells had inhibited expression of the surface protein (Figure 3).

Cell ELISA was a useful technique for the quantitative analysis of cell surface antigen expression and was a sensitive, simple and reproducible method for detecting the cell membrane antigen processing machinery components of cells. Inhibition to target the gene expression of porcine integrin $\alpha \mathrm{v}$ subunit, the FMDV receptor, was performed to demonstrate that the reactivity of pAb located in the membrane of cells was significantly inhibited (Figure 4).
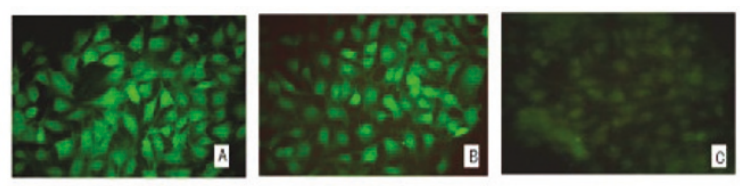

Figure 3 Visualization of A. Mock (+++); B. Control $(+++)$; and C. i $\boldsymbol{\alpha} \mathbf{v}-\mathbf{P K}-15(+)$, by IIF. Samples were incubated with primary antibody (rabbit $\alpha$ v integrin, 1:500) with mouse anti-rabbit IgG-FITC (1:200). Negative (-), no fluorescent cells; weakly positive $(+)$, the signal was faint (less than $5 \%$ of cells); positive $(++), 5 \%$ to $50 \%$ were readily detected; strongly positive $(+++)$, positive staining was intense in more than $50 \%$ of cells. 


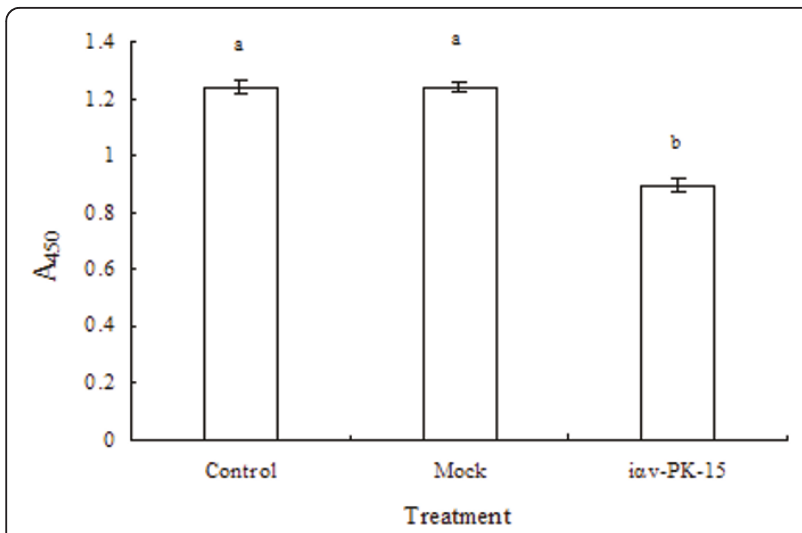

Figure 4 Expression effects of integrin $\alpha v$, as detected by cell ELISA method. Cells $\left(1.0 \times 10^{5}\right)$ were incubated with the isotypematched pAb ( $\alpha$ v integrin, 1:500) with peroxidase-conjugated mouse anti-rabbit lgG (1:10 000), and TMB. Error bars indicate standard deviations. There was a highly significant difference between the control and mock, and iav-PK-15 $(P<0.01)$.

\section{Inhibition of FMDV O/CHA/99 replication in i $\alpha v-P K-15$ cells}

The antiviral activity was tested using cell monolayers in 96-well plates, where $100 \alpha \mathrm{L}$ of the cell suspension $(1.0 \times$ $10^{5}$ cells in each well of a 96-well culture plate) was incubated in $5 \% \mathrm{CO}_{2}$, at $37^{\circ} \mathrm{C}$, allowing at least $24 \mathrm{~h}$ for cells to attach. Cells were infected with $100 \mu \mathrm{l}$ of 100 TCID $_{50}$ FMDV O/CHA/99 and observed microscopically after 48 h. Viral infection caused a marked cytopathic effect (CPE) culminating in total cellular detachment, rounding up, and destruction, which was observed by microscopy [21]. The virus in the supernatant was titrated for infectivity using i $\alpha \mathrm{v}-\mathrm{PK}-15$ cells. After $48 \mathrm{~h}$, viral titers decreased from $10^{4.39}, 10^{4.41}$ in control and mock to $10^{1.29}$ TCID $_{50}$ in i $\alpha v-P K-15$ cells. SiRNAs inhibited virus yield by greater than 1000-fold and there was a > 99\% reduction in virus titer (Figure 5).

\section{Discussion}

FMDV is the prototypic member of the Aphthovirus genus of the Picornaviridae family. The viral capsid is made up from 60 copies each of four viral encoded proteins, VP1 to VP4: VP1, VP2 and VP3 form the outer clasped shell; and VP4 makes up the interior surface [22]. FMDV enters the cell by receptor-mediated endocytosis, in a process that begins with the initial attachment of the virus to cell-surface receptors. The G-H loop forms a major antigenic site on the virion, which includes at its apex an Arg-Gly-Asp (RGD) motif [23,24], a sequence that has been implicated in receptor binding on the basis of synthetic peptide inhibition studies [25]. FMDV can enter cells using different pathways; the main pathway is by binding integrins via a conserved RGD located within the GH loop of VP1 [26,27]. One subgroup within the integrin family is the $\alpha \mathrm{v}$ integrin, which recognizes RGD

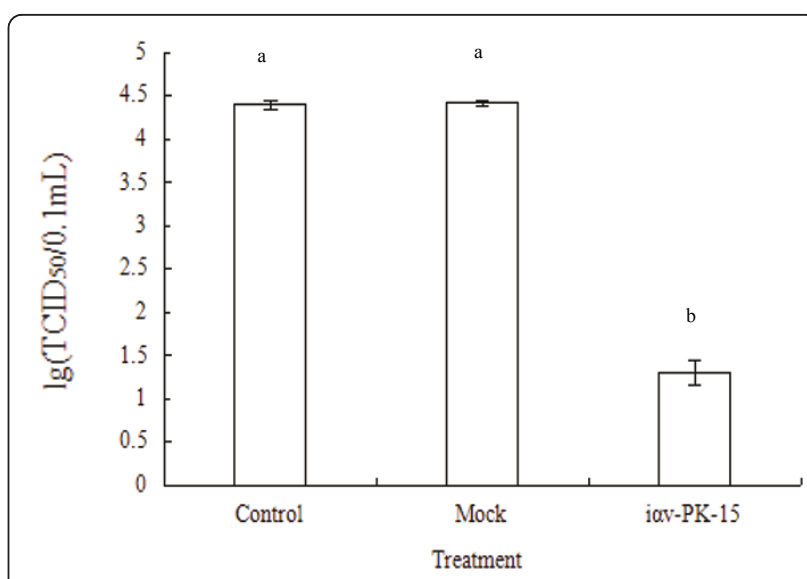

Figure 5 Decrease in virus yield of i $\alpha v-P K-15$ cells. Cells (control, mock, and iav-PK-15) were infected with $100 \mathrm{TCID}_{50}$ of FMDV O/ $\mathrm{CHA} / 99$ per $0.1 \mathrm{ml}$. The virus titer $\left(\mathrm{TCID}_{50}\right)$ was determined three times. Error bars indicate standard deviations. There was a highly significant difference between the control and mock, and iav-PK-15 $(P<0.01)$.

as a binding motif on natural ligands. The $\alpha \mathrm{v}$ subunit is subdivided into two groups based on certain structural differences [28]. The first group is formed by $\alpha-1, \alpha-2, \alpha-$ $L, \alpha-M$, and $\alpha-X$. The second group is formed by $\alpha-3, \alpha-$ $5, \alpha-6, \alpha-7, \alpha-8, \alpha-\mathrm{IIb}, \alpha-\mathrm{V}$ and $\alpha^{\mathrm{IEL}} .24$ functional integrins have been identified in mammalian species, which result from different pairings among $18 \alpha$ subunits and 8 $\beta$ subunits [29]. Different integrins have roles as FMDV receptors $(\alpha v \beta 1, \alpha v \beta 3, \alpha v \beta 6, \alpha v \beta 8)$ [30]. Members of this group all undergo post-translational cleavage of their precursor into a heavy and a light chain. The light chain is composed of the cytoplasmic domain, the transmembrane region, and a part of the extracellular domain (about $25 \mathrm{kDa}$ ), whilst the heavy chain contained the rest of the extracellular domain (about $120 \mathrm{kDa}$ ) [31].

RNAi is a powerful gene silencing mechanism operating in most eukaryotic cells [32]. The effector molecules comprise short duplex RNA sequences of 21-23 bp that direct inhibition of homologous genes. This natural pathway is important for processing of regulatory micro RNAs (miRs) [33]. FMDV is antigenically highly variable and consists of seven serotypes and multiple subtypes [34] that underlie the rapid spreading nature of the clinical disease as early as 2-3 days post-exposure. This means that there is a need to develop new tools that provide early protection, thereby containing the disease spread. RNAi is used as an antiviral technology and has been widely studied for its affect on FMDV $[9,35,36]$. A multiple-siRNA expression system was developed that focused on several regions of the viral genome [37]. Focusing on the conserved regions of the viral genome $[38,39]$, or simultaneously targeting several viral sequences are different approaches to resolving this issue. The multiple-siRNA-expressing plasmid, the pCWN11 
system, is a feasible strategy to overcome the issue of high genetic variability in FMDV and viral escape $[40,41]$. This approach may be used to deal with several viruses, which would be especially useful in the treatment of coinfections by multiple pathogens [8]. Current measures for the control of FMD outbreak include routine vaccination, control of animal movement, and slaughter. FMD vaccines based on inactivated virus and adjuvants are effective in eliminating the disease, but risk the escape of live viruses from animal facilities or as a consequence of improper vaccine preparations $[33,42]$. The development of a recombinant peptide vaccine [43] and a synthetic peptide vaccine [44]) both of which are safe and effective, has been reported. The limited effectiveness of existing vaccines and antiviral drugs means the development of new strategies is essential. The RNAi approach has been reported as an ideal tool to inhibit infectious virus replication in host cells, because siRNA can target and silence important viral genes. However, RNA viruses are likely to evolve mechanisms to suppress or escape an RNAi response. Long-term silencing of viral protein expression by siRNAs has been reported to result in the emergence of viruses resistant to RNA interference $[40,45]$. In particular, integrin $\alpha v$-heterodimers have been previously shown to mediate cellular entry of field strains of the virus. In contrast, cell surface expression of $\alpha \beta 3$ or mRNA for the $\alpha \beta 1, \alpha \beta 3$ or $\alpha \beta 5$ subunits did not appear to contribute to sensitivity of cells to FMDV [46]. We constructed an RNAi lentiviral vector to inhibit target gene expression of the porcine integrin $\alpha \mathrm{v}$ subunit, the FMDV receptor.

It was previously shown that siRNAs inhibited virus yield by 10 -fold to 1000 -fold. The level of viral replication (IND 63/72) inhibition varied depending on the siRNAs produced by the Cocktail Kit. Here, the maximum inhibition (120-fold reduction in virus titer) was observed with the $2 \mathrm{~A}-2 \mathrm{C}$ product compared with both VP3-VP1 (100fold reduction in virus titer) and 3D-3' UTR (40-fold reduction in virus titer) at $24 \mathrm{~h}$. In this case, there was a $>99 \%$ reduction in virus titer with all the serotypes up to 24 h [47]. pCWN11 was a plasmid constructed to express siRNAs with multiple-targeting of the VP1 genes in FMDV. To investigate the effect of pCWN11 on FMDV replication, approximately $10^{3} \mathrm{TCID}_{50}$ of $\mathrm{HKN} / 2002$ virus progeny was detected in the supernatant and collected from cells transfected with pCWN11 at $19 \mathrm{~h}$ p.i. Over $10^{5} \mathrm{TCID}_{50}$ of virus was determined in the supernatant collected from control cells [8]. However, a 1000fold reduction was found in virus titer $24 \mathrm{~h}$ post-infection of BHK cells expressing small hairpin RNA (shRNA) under the control of a mouse U6 promoter from a plasmid construct [35]. We observed a significant cross-inhibition of FMDV replication in BHK-21 cells by siRNAs targeted at various conserved regions of the viral genome (5VNCR, VP4, VPg, POL, and 3VNCR). The results showed that siRNAs generated in vitro by human recombinant dicer enzyme resulted in a viral yield inhibition of 10 -fold to 1000-fold in both homologous (HKN/2002) and heterologous (CHA/99) isolates of FMDV serotype $\mathrm{O}$ at $48 \mathrm{~h}$ post-infection [13]. A sequence corresponding to the critical $30 \mathrm{bp}$ region that forms a stem-loop structure in IRES was also selected for investigation. The regions selected for shRNA were designated as D1, D2, D3, and D4. The virus in the supernatant was titrated in BHK-21 cells to determine infectivity. At 12,18 , and $24 \mathrm{~h}$, the D4-transfected cells yielded a viral titer $\left(10^{-5}\right.$ $\mathrm{TCID}_{50}$ ) that was almost equal to the control. The virus titer in the D2- and D3-transfected cells was less than $10^{-2}$ TCID $_{50}$ at 18 and $24 \mathrm{~h}$. D1-transfected cells yielded $10^{1.4}, 10^{1.7}$, and $10^{2.9} \mathrm{TCID}_{50}$ at 12,18 , and $24 \mathrm{~h}$, respectively [48]. Kahana et al. demonstrated $100 \%$ inhibition of virus growth in BHK-21 cells transfected with a mixture of several anti-FMDV siRNAs [49]. In this study, there was reduction in virus titer of greater than 1000 -fold in i $\alpha v-\mathrm{PK}-15$ cell for O/CHA/99. The results indicate that the reduction in virus titer was either better than the previous study or showed the same effect.

In this study, we constructed an RNAi lentiviral vector containing the packaged i $\alpha$ v-pLenti6/BLOCK-iTTM-DEST expression construct to inhibit target gene expression of the porcine integrin $\alpha \mathrm{v}$ subunit, the FMDV receptor. We co-transfected the ViraPowerTM Packaging Mix and the i $\alpha \mathrm{v}$ pLenti6/BLOCK-iT ${ }^{\mathrm{TM}}$-DEST expression construct containing the U6 RNAi cassette into 293FT cells to produce a replication-incompetent lentivirus, which was transduced into the mammalian cell line of interest. Once the lentivirus enters the target cell the viral RNA is reverse-transcribed, actively imported into the nucleus, and stably integrated into the host genome. The lentiviral construct contains a deletion in the 3' LTR that leads to self-inactivation of the lentivirus after transduction into mammalian cells. Once the lentiviral construct has integrated into the genome, the lentivirus can no longer produce packageable virus and the shRNA of interest is constitutively expressed allowing the performance of transient RNAi analysis, or blasticidin selection to generate a stable cell line for long-term knockdown studies. To determine whether this system can be used to inhibit gene expression of the integrin $\alpha \mathrm{v}$ subunit gene, the expression construct and packing plasmid were transduced into PK15 cells, and we then established an iav-PK-15 cell line. We found that co-transduction resulted in a significant reduction in the corresponding integrin $\alpha \mathrm{v}$ subunit transcription by real-time qRT-PCR, and integrand $\alpha \mathrm{v}$ subunit expression by cell ELISA and IIF. Cell ELISA is a useful technique for the quantitative analysis of cell surface antigen expression, which was developed on the basis of enzyme immunohistochemistry (EIH) and ELISA. Since its development, which was facilitated by the establishment of 
monoclonal antibody technology, a wide range of cell types and surface molecules have been analyzed by cell ELISA, and other methods for the quantitative detection of cell surface molecules. Cell ELISA is not appropriate for analyzing mixed-cell populations. In cell ELISA, labeling of immunoreactant molecules is achieved using an enzyme, such as horseradish peroxidase (HRP). IIF is a rapid sensitive method, which uses cell culture for the detection of integrin $\alpha \mathrm{v}$ subunit expression in cell surface. At present, there is no information available; further work is needed to test the relationship between anti-FMDV efficiency and integrand $\beta 1, \beta 3, \beta 6$ and $\beta 8$.

\section{Conclusion}

Our results suggest that the interference integrin $\alpha \mathrm{v}$ subunit expressing system is a feasible strategy for overcoming the issue of high genetic variability of FMDV, and viral escape. This approach may be developed to combat several viruses, which would be especially useful in the treatment of co-infections by FMDV. In addition, the new strategy would be beneficial in combating viruses with a high viral mutation rate. Our study provides a foundation for the cultivation of new varieties of transgenic organisms. However, this work represents a significant advancement, describing a new approach to trigger anti-FMDV pathways through inhibiting the FMDV receptor gene expression of the porcine integrin $\alpha \mathrm{v}$ subunit.

\section{Acknowledgements}

This work was supported by the National Major Special Project on New Varieties Cultivation for Transgenic Organisms (2009ZX08006-002B, 2009ZX08007-008B and 2008ZX08011-004).

\section{Authors' contributions}

$J D, S G$ and $J$ participated in planning of the study. $J L$ carried out the majority of the experiments and drafted the manuscript. HC conceived the study and helped to draft the manuscript. GZ and JS produced the construction vector. GC, JS and TL participated in cell and virus cultures. All authors read and approved the final manuscript.

\section{Competing interests}

The authors declare that they have no competing interests.

Received: 7 June 2011 Accepted: 7 September 2011

Published: 7 September 2011

\section{References}

1. Bachrach HL: Foot-and-mouth disease virus. Annu Rev Microbiol 1968, 22:201-224.

2. Bass BL: Double stranded RNA as a template for gene silencing. Cell 2000, 101:235-238.

3. Bernstein E, Caudy AA, Hammond SM, Hannon GJ: Role for a Bidentate Ribonuclease in the Initiation Step of RNA Interference. Nature 2001, 409:363-366.

4. Ketting RF, Fischer SE, Bernstein E, Sijen T, Hannon GJ, Plasterk RH: Dicer Functions in RNA Interference and in Synthesis of Small RNA Involved in Developmental Timing in C. elegans. Genes Dev 2001, 15:2654-2659.

5. Hammond SM, Bernstein E, Beach D, Hannon GJ: An RNA-Directed Nuclease Mediates Genetic Interference in Caenorhabditis elegans. Nature 2001, 404:293-296.
6. Nykanen A, Haley B, Zamore PD: ATP Requirements and Small Interfering RNA Structure in the RNA Interference Pathway. Cell 2001, 107:309-321.

7. Ambros V, MicroRNAs: Tiny Regulators with Great Potential. Cell 2001, 107:823-826.

8. Wei Cong, Shaogiang Cui, Jiulian Chen, Xiaoping Zuo, Yonggan Lu, Weiyao Yan, Zhaoxin Zheng: Construction of a multiple targeting RNAi plasmid that inhibits target gene expression and FMDV replication in BHK-21 cells and suckling mice. Vet Res Commun 2010, 34:335-346.

9. Ke Lv, Yingjun Guo, Yiliang Zhang, Kaiyu Wang, Ka Li, Yan Zhu, Shuhan Sun: Transient inhibition of foot-and-mouth disease virus replication by siRNAs silencing VP1 protein coding region. Research in Veterinary Science 2009, 86:443-452.

10. Grubman M, Santos T: Rapid control of foot-and-mouth disease outbreaks: is RNAi a possible solution? TRENDS in Immunology 2005, 26:65-68.

11. Weizao Chen, Mingqiu Liu, Ye Jiao, Weiyao Yan, Xuefeng Wei, Jiulian Chen, Liang Fei, Yang Liu, Xiaoping Zuo, Fugui Yang, Yonggan Lu, Zhaoxin Zheng: Adenovirus-Mediated RNA Interference against Foot-andMouth Disease Virus Infection both In Vitro and In Vivo. JOURNAL OF VIROLOGY 2006, 3559-3566.

12. Francisco Martínez-Azorín, Miguel Remacha, Encarnación Martínez-Salas, Juan PGBallesta: Internal translation initiation on the foot-and-mouth disease virus IRE is affected by ribosomal stalk conformation. FEBS Letters 2008, 582:3029-3032.

13. Mingqiu Liua, Weizao Chena, Zheng Ni, Weiyao Yana, Liang Fei, Ye Jiaoa Jun Zhang, Qingyun Du, Xuefeng Wei, Jiulian Chen, Yumei Liuc, Zhaoxin Zhenga: Cross-inhibition to heterologous foot-and-mouth disease virus infection induced by RNA interference targeting the conserved regions of viral genome. Virology 2005, 336:51-59.

14. Richard O, Hynes: Targeted Mutations in Cell Adhesion Genes: What Have We Learned from Them? DEVELOPMENTAL BIOLOGY 1996, 180:402-412.

15. Gardner $H$, Kreidberg J, Koteliansky $V$, Jaenisch R: Deletion of integrin a1 by homologous recombination permits normal murine development but gives rise to a specific deficit in cell adhesion. Dev Biol 1996, 175:301-313.

16. Hirsch E, Gullberg D, Balzac F, Altruda F, Silengo L, Tarone G: av Integrin subunit is predominantly located in nervous tissue and skeletal muscle during mouse development. Dev Dyn 1994, 201:108-120.

17. Yamada S, Brown KE, Yamada KM: Differential mRNA regulation of integrin subunits av, $\beta 1, \beta 3$, and $\beta 5$ during mouse embryonic organogenesis. Cell Adhesion Commun 1995, 3:311-325.

18. Ji-huai LUO, Jun-zheng DU, SHan-dian GAO, Guo-feng ZHANG, Huiyun CHANG: The optimum siRNA screening of targeting porcine integrin av subunit gene as FMDV receptor against FMDV replication. Act Veterinaria et Zootechnica Sinica 2011.

19. Junzheng Du, Huiyun Chang, Shandian Gao, Shuang Xue, Guozheng Cong, Junjun Shao, Tong Lin, Zaixin Liu, Xiangtao Liu, Xuepeng Cai: Molecular characterization and expression analysis of porcine integrins av $\beta 3, a v \beta 6$ andav $\beta 8$ that are potentially involved in FMDV infection. Molecular and Cellular Probes 2010, 24:256-265.

20. Reed $\amalg$, Muench HA: A simple method of estimating fifty percent end points. Am J Hyg 1938, 27:493-497.

21. Dumbell KR, Jarrett JO, Mautner V, Minson AC, Pereira HG, Pereira MS, Porterfield JS, Wilkinson PJ: Picornaviridae in Porterfield JS (ed) Andrewes' viruses of vertebrates. Cambridge University Press, Cambridge, United Kingdom; 5 1989, 120-145.

22. Manson PW, Grubman MJ, Baxt B: Molecular basis of pathogenesis of FMDV. Virus Res 2003, 91:9-32.

23. Burman A, Clark S, Abrescia NG, Fry EE, Stuart DI, Jackson T: Specificity of the VP1 GH loop of foot-and-mouth disease virus for alpha-v integrins. $J$ Virol 2006, 80:9798-9810.

24. Fry EE, Stuart DI, Rowlands DJ: The structure of foot-and-mouth disease virus. Curr Top Microbiol Immunol 2005, 288:71-101.

25. Alcalá P, Feliu JX, Arís A, Villaverde A: Efficient accommodation of recombinant, foot-and-mouth disease virus RGD peptides to cell-surface integrins. Biochem Biophys Res Commun 2001, 285:201-206.

26. Dicara D, Burman A, Clark S, Berryman S, Howard MJ, Hart IR, Marshall JF, Jackson T: Foot-and-mouth disease virus forms a highly stable, EDTAresistant complex with its principal receptor, integrin av $\beta 6$ : implications for infectiousness. J Virol 2008, 82(3):1537-1546. 
27. Jackson T, King AMQ, Stuart DI, Fry E: Structure and receptor binding. Virus Res 2003, 91:33-46.

28. Ruiz-Sáenz J, Goez Y, Tabares W, López-Herrera A: Cellular Receptors for Foot and Mouth Disease Virus. Intervirology 2009, 52:201-212.

29. van der Flier Sonnenberg: A Function and interactions of integrins. Cell and Tissue Research 2001, 305:285-298.

30. Takada Y, Ye X, Simon S: The integrins. Genome Biol 2007, 8:215.

31. Humpries MJ: Integrin structure. Biochem Soc Trans 2000, 28:311-339.

32. Aagaard L, Rossi JJ: RNAi therapeutics: principles, prospects and challenges. Adv Drug Deliv Rev 2007, 59:75-86.

33. Vreeswijk J: Developments in foot-and-mouth disease vaccines. Vaccine 1991, 9:75-88

34. Grubman MJ, Baxt B: Foot and mouth disease. Clinical Microbiology Reviews 2004, 17:465-493.

35. Chen W, Yan W, Du Q, Fei L, Liu M, Ni Z, Sheng Z, Zheng Z: RNA interference targeting VP1 inhibits foot-and-mouth disease virus replication in BHK-21 cells and suckling mice. J Virol 2004, 78:6900-6907.

36. Mohapatra JK, Sanyal A, Hemadri D, Tosh C, Kumar RM, Bandyopadhyay SK: Evaluation of in vitro inhibitory potential of small interfering RNAs directed against various regions of foot-and-mouth disease virus genome. Biochem Biophys Res Commun 2005, 329:1133-1138.

37. Nishitsuji H, Kohara M, Kannagi M, Masuda T: Effective suppression of human immunodeficiency virus type 1 through a combination of shortor long-hairpin RNAs targeting essential sequences for retroviral integration. J Virol 2006, 80:7658-7666.

38. Chang $\sqcup$, Liu X, He J: Lentiviral siRNAs targeting multiple highly conserved RNA sequences of human immunodeficiency virus type 1 . Gene Ther 2005, 12:1133-1144.

39. Dave RS, Pomerantz RJ: Antiviral effects of Human Immunodeficiency Virus type 1-specific small interfering RNAs against targets conserved in select neurotropic viral strains. J Virol 2004, 78:13687-13696.

40. Gitlin L, Stone JK, Andino R: Poliovirus escape from RNA interference: short interfering RNA-target recognition and implications for therapeutic approaches. J Virol 2005, 79:1027-1035.

41. Geisbert TW, Hensley LE, Kagan E, Yu EZ, Geisbert JB, Daddario-DiCaprio K, Fritz EA, Jahrling PB, McClintock K, Phelps JR, Lee ACH, Judge A, Jeffs LB, MacLachlan I: Postexposure protection of guinea pigs against a lethal Ebola virus challenge is conferred by RNA interference. J Infect Dis 2006, 193:1650-1657.

42. King AM, Underwood BO, McCahon D, Newman JW, Brown F: Biochemical identification of viruses causing the outbreaks of foot-and-mouth disease in the UK. Nature 1981, 293:479-480.

43. Li G, Chen W, Yan W, Zhao K, Liu M, Zhang J, Fei L, Xu Q, Sheng Z, Lu Y, Zheng Z: Comparison of immune responses against foot-and-mouth disease virus induced by fusion proteins using the swine IgG heavy chain constant region or $\beta$-galactosidase as a carrier of immunogenic epitopes. Virology 2004, 328:274-281.

44. Wang CY, Chang TY, Walfield AM, Ye J, Shen M, Chen SP, Li MC, Lin YL, Jong MH, Yang PC, Chyr N, Kramer E, Brown F: Effective synthetic peptide vaccine for foot-and-mouth disease in swine. Vaccine 2002, 20:2603-2610.

45. Das AT, Brummelkamp TR, Westerhout EM, Vink M, Madiredjo M, Bernards R, Berkhout B: Human Immunodeficiency Virus type 1 escapes from RNA interference-mediated inhibition. J Virol 2004, 78:2601-2605.

46. Donald PK, Alison B, Sarah G, Andrew ES, Terry J, Nigel P, Ferris : Integrin sub-unit expression in cell cultures used for the diagnosis of foot-andmouth disease. Veterinary Immunology and Immunopathology 2011, 140:259-265

47. Jajati Keshari Mohapatra, Aniket Sanyal, Divakar Hemadri, Chakradhar Tosh Manoj Kumar R, Santanu Kumar Bandyopadhyay: Evaluation of in vitro inhibitory potential of small interfering RNAs directed against various regions of foot-and-mouth disease virus genome. Biochemical and Biophysical Research Communications 2005, 329:1133-1138.

48. Joyappa DH, Sarika Sasi, Ashok KC, Reddy GR, Suryanarayana WS: The plasmid constructs producing shRNA corresponding to the conserved 3D polymerase of Foot and Mouth Disease virus protects guinea pigs against challenge virus. Vet Res Commun 2009, 33:263-271.

49. Kahana RL, Kuznetzova A, Rogel M, Shemesh D, Hai H, Yadin YStram: Inhibition of foot-and-mouth disease virus replication by small interfering RNA. J Gen Virol 2004, 85:3213-3217.
doi:10.1186/1743-422X-8-428

Cite this article as: Luo et al:: Lentviral-mediated RNAi to inhibit target gene expression of the porcine integrin $\alpha \mathrm{v}$ subunit, the FMDV receptor, and against FMDV infection in PK-15 cells. Virology Journal 2011 8:428.

\section{Submit your next manuscript to BioMed Central and take full advantage of:}

- Convenient online submission

- Thorough peer review

- No space constraints or color figure charges

- Immediate publication on acceptance

- Inclusion in PubMed, CAS, Scopus and Google Scholar

- Research which is freely available for redistribution 